\title{
Understanding Brain Plasticity in Learning Process
}

\author{
Yuliana Yuliana \\ Anatomy Department, Medical Faculty, Udayana University, Indonesia \\ lee.yuliana@gmail.com
}

\begin{abstract}
Brain plasticity takes an essential part in the learning process and memory retaining. Brain plasticity is connected with myelin sheath development and oligodendrocyte plasticity. Dynamic myelination is attained through certain learning activities. Oligodendrocyte plasticity means changes of oligodendrocyte precursor cell number either increasing or decreasing. This review aims to reveal what learning style is better in optimizing learning capacity by understanding dynamic myelination, brain plasticity, oligodendrocyte plasticity, and learning process. The review paper was based on journal reading. Keywords for literature searching are "Learning, myelin, brain, plasticity, oligodendrocyte" with filters in the last 5 years in the PubMed website. Pubmed was considered as one of the qualified journals. It doesn't rule out the possibility of using another journal that is not listed in PubMed. Result showed 36 articles. Other citations were added based on the previously selected journals. The learning process is closely related to encoding. Making schema while learning new objects are very useful in increasing oligodendrocytes cells number. The paper limitation is it didn't describe how the learning process might improve school performance and success in later life. There isn't any comparison about how each learning activity has an unequal effect on dynamic myelination, brain plasticity, and daily life skills improvement. This review paper's significance is myelin was not static, but it is dynamically changed through learning activities, new experiences, musical, or even practical training. Exposure to a new environment in the learning process will improve myelination and optimize learning. Learning new things is good for our brain because it will prevent dementia.
\end{abstract}

Keywords:

Learning; myelin; brain; plasticity

This is an open access article under the CC-BY-NC license.

\section{INTRODUCTION}

Learning is an important part of our daily life. We learn how to drink, eat, crawl, walk, run, and even memorize many things. Playing musical instruments, riding a bike, playing football also need learning as important activities. Learning involves many parts of our body. They are limbs, brain, sensory, and reflex. The brain is the coordinator of other parts of our body. Learning can't be separated from the brain. The brain has plasticity property. Learning has an important contribution to brain plasticity. To learn is to myelinate. Myelination is equal to brain plasticity. Learning might change white matter microstructure and myelin sheaths number (Arancibia-Carcamo et al., 2017).

Brain cell, namely neuron, is covered by a myelin sheath. Oligodendrocytes and Schwann cells form the myelin sheath. Myelination is dynamically changing from time to time (Mount \& Monje, 2017). Understanding how dynamics of 
myelination and brain plasticity will Kato 2015). Oligodendrocytes are optimize learning capacity. This review categorized as multipolar giant cells (Fields, aims to reveal what learning style is better 2015).

in optimizing learning capacity by understanding dynamic myelination and brain plasticity (Long and Corfas, 2017).

This review aims to reveal what learning style is better in optimizing learning capacity by understanding dynamic myelination, brain plasticity, oligodendrocyte plasticity, and learning process. The learning process is closely related to encoding. Making schema while learning new objects are very useful in increasing oligodendrocytes cells number. This review paper's significance is myelin is dynamically changed through learning activities, new experiences, musical, or even practical training. A new environment or new habit in the learning process will improve myelination and optimize learning.

\section{THEORETICAL PERSPECTIVES}

Information processing takes place in the gray matter of the brain. Synapses connect different neurons. They reside in the gray matter. Synapses are the major components of information processing and plasticity. Myelinated axons lie beneath the gray matter and form functional neuronal circuits. Myelin formed by oligodendrocytes shows plastic changes depending on neuronal activity (Wake \&
Brain processes information depend on the action potential. These action potential are affected by tripartite synapse consists of astrocytes or oligodendrocyte. Oligodendrocyte takes an important part in axonal myelination (Yamazaki et al., 2018). Impuls delivery will be enhanced along the axons (Cabezas et al., 2017). Myelination is very important in rapid saltatory conduction. Myelinated axons will deliver information faster than unmyelinated ones (Williamson and Lyons, 2018). Action potential velocity will be dramatically increased (Yamazaki, 2019). Cognitive development will be increased. We can think and move faster due to myelin sheath involvement (Arancibia-Carcamo et al., 2017). Axonal conduction (included plastic changes) are occurred due to oligodendrocyte depolarization. Oligodendrocytes and astrocytes are also called perineuronal glial cells (Yamazaki et al., 2018).

It was revealed in a study that there was a synapse between myelin and axon. Axon might dynamically signal glia. Axomyelinic synapse might support the learning process. Myelin Whilst disorder in the synapse will cause abnormalities of the central nervous system such as 
schizophrenia and multiple sclerosis (Micu et al., 2016).

Perineuronal oligodendrocytes can depolarize or hyperpolarize based on input stimulation, thus these cells are important in neuronal activity modulation. Neuron afterhyperpolarization might be increased by aging, fear, or eye blink learning. Meanwhile, decreasing of afterhyperpolarization could happen during learning (Yamazaki et al., 2018).

Myelin was previously considered as a static electric insulator, however recent studies reveal that myelin can be dynamically regulated by neuronal activity (Fields, 2015). Myelin takes an important part in brain plasticity. Enriched environments and experiences might increase myelination (Kaller et al., 2017; Swire \& Constant, 2018). In a study, there was a delayed loss of white matter when the enriched environment was given. Myelin volume and length also increased in the experimental group which consisted of middle age and old age rats (Fields, 2015). Myelin is formed in a special place between segments of the Ranvier node. Ranvier node (node of Ranvier) is a special segment that is unmyelinated. Myelin is important in increasing the conduction velocity of impulse propagation along axons. Impulses will be delivered faster by going through myelinated axons (Freeman et al., 2016).
Myelin is very complex in structure and formation compared with synapse. Myelin is the most complex intercellular junction. It is the largest among the other cellular junctions. Myelin formation needs harmonay of biological cell processes to recognize cell and membrane in a specific way, to wrap axons in smooth order, and maintain appropriate ion channel throughout the life span. Myelin and Ranvier node can only be seen through electron microscopy. However, myelin has a large size compared to other cells in the brain. Myelin plasticity involves behavioral, molecular, morphological, and electrophysiological changes (Fields, 2015). Activity-based on myelination is very critical in a larger brain due to complex neural activity. Conduction delay and precise brainwave rhythm are two substantial items in a larger brain. Myelin plasticity is crucial in the learning process. It will enhance cognitive performance. Any abnormalities in this process will cause neuropsychiatric and neurological disorders. Understanding the process precisely might suggest new insight in seeking the best therapy for related disorders (Fields, 2015).

Memory is dynamic. It is being stored and encoded in a continuous process. The brain is very capable of creating meaning. Creating new meaning in the orderly pattern 
of preexisting knowledge structures called schemas (Hasan et al., 2019).

Hippocampus is involved in various emotional, cognitive, and sensory information (Hasan et al., 2019). Long-term memories consolidation including declarative and autobiographical memories are processed in the hippocampus. Gene expression is important in changing new information into long-lasting memories. Long-term structural change will be formed at the end of the process of consolidation (Travaglia et al., 2016).

\section{METHODOLOGY}

This review paper was based on journal reading. Keywords for literature searching are "Learning, myelin, brain, plasticity, and oligodendrocyte" with filter published in the last 5 years in the PubMed website. Result showed 36 articles. Those 36 journals were selected based on their relevancies to the paper topic. Other articles in the references were added based on the previously selected journals. Pubmed was considered one of the qualified journals. It doesn't rule out the possibility of using another journal that is not listed in PubMed.

\section{FINDINGS AND DISCUSSION}

Myelin sheath regulates central nervous system plasticity and repair. These special sheath changes contribute to the fine-tuning of neuronal networks. Thus, it will affect the nervous system plasticity, cognitive processing, and learning (Jeffries et al., 2016). In the end, myelin plasticity is crucial in the learning process. Activitydependent changes in myelin result in many signals on the spatial and temporal aspects (Gibson et al., 2017).

Myelin plasticity needs a cell to cell recognition. There is a special signal complex among axon, neuron cell, and glial cells. Electrical activity in axons arranges all phases of myelination. The phase starts when oligodendrocyte signals an axon to make a special intercellular junction. The electrical stimulation of axons increases intracellular calcium (Fields, 2015).

Learning style

Comparison actions are considered as low-demanding tasks. Prolonged unchallenging activities might worsen cognition and brain function. Therefore, learning should include new skill mastery or an environment to optimize its capacity (Baddeley et al., 2017). Learning new skills might include learning how to play the piano, running, doing juggling, or even reaching exercise (Arancibia-Carcamo et al., 2017; Field, 2015).

Learning changes adult white matter. New myelin-forming oligodendrocytes and myelinated axons will be created (Bujalka 
\& Emery, 2017). Oligodendrocytes cells and training enlarged anterior corpus coil axon membranes in a circle way to callosum compared to controls. Various form the myelin sheath (Cabezas et al., sports training will increase 2017). Myelin is formed of the fatty oligodendrocyte genesis (Keiner et al., substance (Arancibia-Carcamo et al., 2017). 2017). Running increases synaptic plasticity

Axons function is a transmission line and cellular activity in the hippocampus from one neuron to another by using (Zheng et al., 2019). Synaptic plasticity specific action potential at the neuron means synapse formation (synaptogenesis), proximal part. Action potential velocity remodeling, and elimination (Fields, 2015). might change according to the activity type. Learning new skills might mediate Sometime, axons might fail in their oligodendrocyte development in the function. Axon might express ionotropic and metabotropic receptors, which could induce neuromodulation (Bucher, 2016).

The brain responds to physical exercise, motor learning, social interaction, and the enriched environment through cellular and functional changes. White matter volume will be increased. Sensorymotor stimulation increased more oligodendrocytes in the visual cortex. Meanwhile, sensory deprivation and social isolation will decrease oligodendrocytes number (Keiner et al., 2017; Swire \& Constant, 2018). Social isolation will affect prefrontal cortex changes. Finally, impulse conduction will be decreased (Etxerberria et al., 2016).

Acquiring new motor skills involve memory consolidation and skill learning. somatosensory cortex and motor cortex. Cortical neurons and corpus callosum will also be stimulated. Cell number and thickness of oligodendrocytes might increase about 4 weeks after the stimulation. There will be new myelin sheath formed by oligodendrocytes on the active axons (Xiao et al., 2016). Once new myelin sheaths are formed, they are likely to remain there even though the stimulations are stopped (Hughes et al., 2018).

Mental schema's roles are as cognitive structures. The medial prefrontal cortex is important in processing schema-related mnemonic function. Hippocampus will interact with anterior cingulate cortex to guide decision making through experiences (Hasan et al., 2019).

The more practice is done, the more automatic skills will be achieved (Lakhani et al., 2016). Musical instrument learning 
Brain plasticity

Brain plasticity comprises of myelin plasticity, neuron oligodendrocyte plasticity, plasticity, plasticity, and white matter plasticity. Plasticity is the ability of neural components to reform their shape and connectivity through experience, emotions, cognition, or learning new things through an adaptable manner. Plasticity may positively enhance cognition. The circuit function will be changed through plasticity. Stress might also contribute to plasticity. Elevated plasticity markers might favor the memory and learning process. However, the uncontrolled elevated marker may cause neurologic diseases (Cabezas et al., 2017).

Brain plasticity study focused on a synapse. Synapse is very important in the learning process and memory. Nevertheless, plasticity might happen outside synapses. Plasticity might happen in myelin, the white matter of the brain, and other parts of the brain. Those precious experiences will powerfully shape the brain and result in plasticity (Cabezas et al., 2017).

There are variations of synaptic plasticity among brain regions. The cerebral cortex has systemic variation. The limbic area is more variable in synaptic plasticity than cortical and eulaminate area. Larger dendritic arbors are found much more in the limbic area than the eulaminate area.
Pyramidal neurons are also variable in their myelin content. Synaptic plasticity variation depends on synaptic turnover and postsynaptic receptors (Cabezas et al., 2017). These variabilities might be due to various stages of cell development, different stimulation such as learning type, emotions, or physical condition (ArancibiaCarcamo et al., 2017). Variations of myelinated axons are very useful to adjust their conduction velocity. Changes in membrane capacitance and axial resistance will affect current flow in axons. Internode length in the peripheral nervous system is decreased near the synaptic terminal to make conduction time more effective (Ford et al., 2015).

Astrocytes as glial cells have essential roles in a molecular and cellular process. These cells regulate neurotransmitters and synapse. Thus, synaptic plasticity will be higher (Singh and Abraham, 2017).

White matter is almost half of total brain content. It consists of axons. Axons are available in two types, myelinated and nonmyelinated (unmyelinated). The composition of myelin is mostly lipid. The total length of all myelinated axons of an adult brain is approximately 160,00 kilometers. White matter also consists of oligodendrocytes, microglia, astrocytes, and oligodendrocyte precursor cells. In a study of rats, one oligodendrocyte cell was able to 
myelinate more than 20 axons. One remyelinating of demyelinated axons after example of a disorder of white matter is environmental enrichment (Fields, 2015). multiple sclerosis (Fields, 2015).

Various activities such as sleep, selective

The brain develops well until attention, learning, memory gathering, adulthood and starts to decline gradually emotion, and consciousness are related to around 50 years olds. Experiences, different brain rhythms. Complex neural exercises, sleep, reading, decision making, activity will yield a complex neural vocabulary memorizing, and new skill network. These conditions depend closely learning will cause white matter changes in whole life. Long time practice such as piano playing also causes white matter changes, i.e. in corticospinal tract fibers. Dancing or typing has the same effect on white matter changes too. The onset of training, training frequency, learning strategy, and stages are important factors to be considered in expecting white matter changes result. Greater changes yield better performance. Maturation of oligodendrocyte cells may increase in white matter as a response to complex training (Xiao et al., 2016).

White matter plasticity happened due to memory training or motor learning. The first phenomenon of cell changes due to aging is white matter degradation. It happens in an anterior-posterior gradient. It is related to a decrease in memory. Myelin integrity will be lost during aging. Learning routinely might inhibit the aging process of the brain and maintain better cognitive development. Motor learning will induce the proliferation of oligodendrocytes in the elderly. In aged animal studies, there was on conduction delay and myelination. Conduction delay is very sensitive to fine and subtle changes in conduction velocity. Therefore, myelination and activities are highly bound to one another. Any abnormalities of conduction velocity will cause white matter abnormalities (Fields, 2015).

Exercise might enhance neurogenesis and angiogenesis in the hippocampus. It might be new blood vessels form in the affected part. Hippocampal volume increasing was usually accompanied with myelination, not blood flow increase (Thomas et al., 2016). Physical fitness due to exercise was well correlated with cognitive performance such as spatial memory (Oberlin et al., 2016).

Enough sleep might help in neural network homeostasis. Learning, consolidation of memory, and motor activity depend on enough sleep. Synaptic plasticity may increase. Long term neuronal activity could be well maintained. The proliferation of oligodendrocyte precursor 
cells is increasing during sleeping. conduction velocity in optimizing neural Differentiation of oligodendrocytes is circuits. Myelination process might be dominant in awake time. Therefore, affected by action potentials firing in insomnia and other sleeping disorders myelinated axons (Fields, 2015). might disturb oligodendrocyte precursor cell proliferation. In the end, myelination might be disrupted (Fields, 2015).

One specific mechanism for plasticity is the Hebbian plasticity mechanism. It is long term potentiation as a great substrate for the learning process. Any positive experience such as learning new skills might shape the brain circuit connection. White matter has dynamic plasticity which depends on experience and learning process (Sampaio-Baptista and Johansen-Berg, 2017).

Synaptic plasticity could happen because there is a coincident activity between two neurons to strengthen their connection. Hebbian synapse concept has laid the basis for the theory of learning and plasticity. However, there is a lack of evidence about this mechanism (Fields, 2015).

\section{Mechanisms of Plasticity}

In previous studies, a synapse is the main topic for studying nervous system plasticity in the learning process. However, in recent studies, plasticity is considered a new mechanism. Moreover, plasticity is correlated with myelination remodeling and
Plasticity might take place because of activity, experience, learning, or special mechanism such as functional changes of axons to alter behavior (Sampaio-Baptista and Johansen-Berg, 2017). Meanwhile, any changes in the diameter of myelinating axons or length of internode (nodes of Ranvier) might alter physiological changes such as conduction speed. Changes in conduction speed might change behavior (Field, 2015).

Myelin thickness may differ due to the differentiation of oligodendrocyte precursor cells become new oligodendrocytes. Thus, oligodendrocytes have control in regulating myelin thickness. Newly formed oligodendrocytes and the old oligodendrocytes can remodel myelin. However, experiences, stimulation, or social isolation might change remodeling. Lower brain activity is associated with lower myelination and myelin remodeling (Sampaio-Baptista and Johansen-Berg, 2017). Myelin remodeling means formation, proliferation, differentiation, migration, and survival of glial cells that produce myelin sheath. Biological mechanisms in wrapping axons and the formation of Ranvier nodes are also 
involved in the process of myelin myelinating cells. Satellite remodeling (Fields, 2015).

Approximately $5 \%$ of the total cells are oligodendrocytes have special functions such as metabolic support, apoptosis in oligodendrocyte precursor cell forms. These oligodendrocyte precursor cells are only $10-15 \%$ of the total glial cells. Even though the number is few, these oligodendrocyte precursor cells can form new myelinating oligodendrocytes. Oligodendrocyte precursor cells can spike electric impulses to yield specific signals to guide myelination in axons (SampaioBaptista and Johansen-Berg, 2017).

Neuron-glia interactions

Neuron-glia interactions are crucial in maintaining optimal central nervous system function. Glia cells might modulate potentiation or depression of synapses. Neuronal activity may be maintained in optimal condition if the rhythmic network of glial cells is well regulated. Thus, glial cell arrangement is very essential. Oligodendrocytes are the most cells found in grey matter. Astrocytes and microglia are only found in a small number. Oligodendrocytes reside in grey matter substance are also called perineuronal or satellite oligodendrocytes (Battefeld et al., 2016).

Perineuronal oligodendrocytes (satellite oligodendrocytes) are mostly nonmyelinating. It is very different from the main function oligodendrocytes as protection, remyelinating axons especially in the perisomatic area after injuries, and regulate action potential firing. Although satellite oligodendrocyte was considered as non-myelinating oligodendrocytes, like oligodendrocyte progenitor cells, some findings revealed that satellite oligodendrocytes were able to myelinate axons. Interactions among neuron, astrocyte, and oligodendrocyte are very crucial in axonal myelination and neuronal excitability (Battefeld et al., 2016).

Researchers found that there was an axonal projection increasing in using tools training of monkeys. Branches of axons were growing faster in spatial learning in other studies. Reorganization of axonal boutons rapidly occurred in response to neuronal activity and experiences (Sampaio-Baptista and Johansen-Berg, 2017). Oligodendrocytes need ion channels and neurotransmitter receptors to proceed with their functions. Neurotransmitters released along axons might signal neurotransmitter receptors at the myelinating glia in hypoxia or energy depletion condition (Fields, 2015).

Myelination

Myelination happens from birth throughout life and gradually decreases 
with increasing age (Williamson and Lyons, 2018). Fard et al. (2017) found that after the third decade of life, new oligodendrocyte will be decreased in white matter. Therefore, everyone needs to stimulate neuronal activity by learning new skills continuously. Myelin wrapping changes might be involved in the learning process. Changes in node length, myelin thickness, and myelin length will affect signal velocity transmission. Myelination is very crucial in the proper function of the nervous system (Fields, 2015).

Myelination is rapidly emerging in children. It continues widespread along the axons in a spatiotemporal way. Therefore, children tend to gain cognitive development well in the first few years of their life. However, sometimes there are myelination abnormalities such as in multiple sclerosis disease. It is a demyelinating disease (Williamson and Lyons, 2018).

Variation in number, length, thickness, and distribution of myelin are contributing factors of myelin plasticity. There are many different patterns of myelination. Changes in those contributing factors might affect the speed of impulse conduction. Fine changes in myelination pattern in the form of addition or remodeling, might change impulse conduction time. Therefore, dynamically modifying myelination might promise a cure in a certain case
(Williamson and Lyons, 2018). Increasing new myelinating oligodendrocyte production might be helpful in motor learning (Xiao et al., 2016). Dynamics of myelination could affect neural circuit function (Williamson and Lyons, 2018).

Myelination is critical in connecting the brain by enhancing the speedy transfer of information in the brain and related structures (spinal cord). It is crucial in maintaining the best cognitive performance functions. Any myelin disturbance may cause dysregulations of many neural circuits. Those conditions might produce disease symptoms. Studying myelination comprehensively has an important role in diagnosing and choosing the treatment of these diseases (Nickel and Gu, 2018).

Myelin Regulation as a New Mode of Brain Plasticity

Myelination is continuously happening now and then. Remodeling of myelin continues in adulthood. This process includes myelin remodeling, myelinating axons, and repairing the partially myelinated axons. Therefore, the oligodendrocyte cell number is changing continuously. A new oligodendrocyte is made of oligodendrocyte precursor cell differentiation. Sometimes, the aging process leads to apoptosis of oligodendrocyte cells. Myelin sometime undergoes apoptosis also. Turnover is 
inevitable process. Approximately, 2.5\% of new oligodendrocyte is added annually in the gray matter. However, only about $0.33 \%$ is added to the corpus callosum. By routine myelin remodeling, neural networks will be better. Therefore, brain plasticity takes place (Nickel and Gu, 2018).

Oligodendrocyte precursor cells derived from the neural stem cells. These cells then proliferate and migrate, whilst proliferating and differentiating into oligodendrocyte cells and finally myelinating axons. Locally secreted factors and neuronal activity might impact oligodendrocyte development. Therefore, there are the various degree of myelin plasticity among central nervous system regions. Myelin remodeling induced by learning is revealed in many studies involving animals. Motor skill learning enhanced the production of oligodendrocyte cells. (Nickel and Gu, 2018).

Myelination is begun by oligodendrocyte maturation. Oligodendrocyte progenitor cells will differentiate into oligodendrocyte (Williamson and Lyons, 2018). It is revealed that most of the new oligodendrocyte cells will die. Finally, there is only $22 \%$ of them are survive. Despite these facts, myelinating oligodendrocyte will remain stable until fifty days. It was found that about $81 \%$ of myelin sheaths are stable in early adulthood. Hill et al. (2018) found that middle-age people tend to have stable oligodendrocytes until eighty days. It was also revealed in the research that almost $99 \%$ of sheath were stable in more than three weeks (Hughes et al., 2018).

However, oligodendrocyte number decreases significantly in increasing age. That's why myelin number will decrease also because myelin is made of oligodendrocytes cell. Decreasing myelin number will arise new problems such as cognitive deterioration disorder such as dementia (Ritchie et al., 2015). The microstructure of white matter will vanish with increasing age. Demyelinating axons reduces the efficiency of information transfer. Cortical disconnection will be the result (Cox et al., 2016).

In the ablation of the oligodendrocyte experiment, Auer et al. (2018) studied about myelin sheath damage. They concluded that when one myelin sheath is damaged, the surrounding myelin sheath was able to grow rapidly to wrap the lost part. A certain part of myelination is maintained to hold the specific optimal conduction pattern of the axons. This condition may keep the conduction time consistent from time to time within the neural circuits as the axon lengths change. Such a remodeling pattern might act as a compensatory mechanism of the loss of myelin sheath. Myelin loss 
related to aging may cause remodeling of length could impact conduction speed existing myelin sheath to keep circuit (Hughes et al., 2018).

function consistently flowing. Therefore, most myelin sheath lengths are mostly stable and the circuit function is well regulated throughout life. Myelin length, thickness, and internode length adjustment might be important in controlling the velocity of myelinated axons. These myelin changes might be achieved through new experiences (Auer et al., 2018).

Recently, researchers found that stimulating sensory could enhance oligodendrocyte numbers. This might be due to newly formed differentiated cells. De novo myelination is stimulated through the neural circuit in cortex along with life. Remodeling of myelin might change impulse conduction velocity because every fine change of myelin length could alter the node conduction speed (Hughes et al., 2018).

Length of a node might be regulated by molecules Caspr, contactin, and neurofascin 155 through modifying adhesion of paranodal cell. The regulator of node length is nodal amyloid precursor protein. Changes in those molecules might result in dementia Alzheimer's. Node length modulation may adjust action potential time. This condition usually happens in major depression, chronic stress, or hypoxia. Changes in node
The precise time for the action potential is very crucial in processing the information load. However, the factors contributing to action potential timing haven't fully revealed. Precise stimulation is thought of as a very helpful modal in maintaining the optimal time of action potential and regulating conduction velocity (Ford et al., 2015). Speed and time are key factors in signal transmission optimization. Precision and synchrony are the main focus (Fields, 2015).

A great variation of myelinated axons features has an important role in information processing. However, an additional factor might take a role. The factor is variation in the density of ion channel. Remodeling of the axons might happen in adults. Some parameters adjustment such as length of internode, axon diameter, myelin thickness, and node diameter might tune information processing and affect conduction velocity. Those parameters might change white matter structure in the learning process (Ford et al., 2015). There will be fewer myelinated axons in a complex neural network. This area tends to undergo plasticity in myelination (Sampaio-Baptista and Johansen-Berg, 2017). 
Myelin thickness changes in very helpful in signal coordination. myelination are necessary for adaptive However, there is a tremendous variation of changes in conduction speed along the myelination in every brain region. Fine and learning process. Myelin thickness might be local myelination is very useful in tuning, adjusted for optimal impulse conduction coordinating, and ensuring precise timing of delivery speed in each axon. Changes in the Ranvier node structure might also alter action potentials (Ford et al., 2016; Arancibia Carcamo et al., 2017). conduction speed adaptively (Fields, 2015).

Myelinating of unmyelinated axons is a crucial part in the learning process. This

\section{CONCLUSION}

Optimizing learning capacity can be process will affect neural activity. achieved by understanding dynamic Conduction time will be increased. It will yield a profound effect on neural activity. Finally, myelin plasticity takes place in a learning activity (Fields, 2015).

Learning is better done in early life, whilst myelination is actively happening. Myelination of the prefrontal cortex is growing actively. A recent study revealed that the amygdala and corpus callosum revealed increasing in oligodendrocyte progenitor (precursor) cells while the environment was enriched and exercise was given. Motor skill learning needs long time practice. The longer the practice time, the better myelination will be. Learning showed white matter changes in fornix only in two hours' time in studies using humans and animals. Those changes related to behavioral development. Rapid myelination was found (Fields, 2015).

\section{REFERENCES}

Myelination in a larger brain is very important. Faster conduction velocity is

Arancibia-Cárcamo, I. L., Ford, M. C., Cossell, L., Ishida, K., Tohyama, K., 
and Attwell, D. (2017). Node of Ranvier length as a potential regulator of myelinated axon conduction speed. eLife6: e23329.doi:10.7554/eLife.23329

Auer, F., Vagionitis, S., and Czopka, T. (2018). Evidence for myelin sheath remodeling in the CNS revealed by in vivo imaging. Curr. Biol. 28, 549-559. doi:10.1016/j.cub.2018.01.017

Baddeley, C.M., Foley, S., de Santis, S., Charron, S., Hampshire, A., Caeyenberghs, K., \& Jones, D.K. (2017). Dynamics of White Matter Plasticity Underlying Working Memory Training: Multimodal Evidence from Diffusion MRI and Relaxometry. Journal of Cognitive Neuroscience 29:9, 1509-1520.

Battefeld, A., Klooster, J., Kole, M.H.P. (2016). Myelinating satellite oligodendrocytes are integrated in a glial syncytium constraining neuronal high-frequency activity. Nature Communications|7:11298|DOI:10.1038 /ncomms11298|www.nature.com/natur ecommunications.

Bergles, D.E., Richardson, W.D. (2016). Oligodendrocyte Development and Plasticity. Cold Spring Harb Perspective in Biology. 8: 1-27.

Bucher, D. (2016). Chapter - 12 Contribution of Axons to Short Term
Dynamics of Neuronal

Communication. Axon and Brain Architecture. Page 245-263.

Bujalka, H., \& Emery, B. (2016). Cellular Mechanism of Adaptive Myelination: Bridging the Gap between Animal Studies and Human Cognition. Cogn Neurosci. 8(2):122-124.

Cabezas, G.M.A., Joyce, M.K.P., John, Y.J., Zikopoulos, B., Barbas, H. (2017). Mirror trends of plasticity and stability indicators in primate prefrontal cortex. Eur J Neurosci 46(8): 23922405.

Cox, S. R., Ritchie, S. J., Tucker-Drob, E. M., Liewald, D. C., Hagenaars, S. P., Davies, G., Wardlaw, J.M., Gale, C.R., Bastin, M.E., Deary, I.J. (2016). Ageing and brain white matter structure in 3,513 UK Biobankparticipants. Nat.Commun. 7:13629.doi:10.1038/ncomms13629.

Etxeberria, A., Hokanson, K.C., Dao, D.Q., Mayoral, S.R., Mei, F., Redmond, S.A., Ullian, E.M., and Chan, J.R. (2016). Dynamic modulation of myelination in response to visual stimuli alters optic nerve conduction velocity. J. Neurosci. 36, 6937-6948.

Fard, M. K., Van der Meer, F., Sánchez, P., Cantuti-Castelvetri, L., Mandad, S., Jäkel, S., Fornasiero, E.F., Schmitt, S., Ehrlich, M., Starost, L., Kuhlmann, T., 
Sergiou, C., Schultz, V., Wrzos, C., Bruck, W., Urlaub, H., Dimou, L., Stadelmann, C., Simons, M. (2017).

BCAS1 expression defines a population of early myelinating oligodendrocytes in multiple sclerosis lesions. Sci. Transl.

Med.

9:eaam7816.doi:10.1126/scitranslmed. aam 7816.

Fields, R.D. (2015). A new mechanism of nervous system plasticity: activity dependent myelination. Nat. Rev. Neurosci. 16, 756-767.

Ford, M.C., Alexandrova, O., Cossell, L., Stange-Marten, A., Sinclair, J., KoppScheinflug, C., Pecka, M., Attwell, D., Grothe, B. (2015). Tuning of Ranvier node and internode properties in myelinated axons to adjust action potential timing. Nature Communications|6:8073|DOI:

10.1038/ncomms9073|

www.nature.com/naturecommunication s.

Freeman, S.A., Desmazie` res, A., Fricker, D., Lubetzki, C., and Sol-Foulon, N. (2016). Mechanisms of sodium channel clustering and its influence on axonal impulse conduction. Cell. Mol. Life Sci. 73, 723-735.

Gibson, E.M., Geraghty, A.C., \& Monje, M. (2018). Bad Wrap: Myelin and
Myelin Plasticity in Health and

Disease. Dev Neurobiol. 78(2):123135.

Hasan, M., Kanna, M.S., Jung, W., Ramkrishnan, A.S., Iqbal, Z., Lee, Y., \& Li, Y. (2019). Schema-like learning and memory consolidation acting through myelination. FASEB J. 33(11): 11758-75.

Hill, R.A., Li, A.M., and Grutzendler, J. (2018).

Lifelongcorticalmyelinplasticity and age-related degeneration in the live mammalian brain. Nat. Neurosci. 21, 683-695.

Hughes, E.G., Orthmann-Murphy, J.L., Langseth, A.J., and Bergles, D.E. (2018). Myelin remodeling through experience-dependent

oligodendrogenesis in the adult somatosensory cortex. Nat. Neurosci. 21, 696-706.

Jeffries, M.A., Urbanek, K., Torres, L., Wendell, S.G., Rubio, M.E., \& FyffeMaricich, S.L. (2016). ERK1/2 Activation in Preexisting Oligodendrocytes of Adult Mice Drives New Myelin Synthesis and Enhanced CNS Function. The Journal of Neuroscience. 36(35): 9186-9200.

Kaller, M.S., Lazari, A., Blanco-Duque, C., Sampaio-Baptista, C., \& JohansenBerg, H. (2017). Myelin Plasticity and 
Behaviour-Connecting the Dots. Curr Opin Neurobiol. 47:86-92.

Keiner, S., Niv, F., Neumann, S., Steinbach, T., Schmeer, C., Hornung, K., Schlenker, Y., Förster, M., Witte, O.W., \& Redecker, C. (2017). Effect of skilled reaching training and enriched environment on generation of oligodendrocytes in the adult sensorimotor cortex and corpus callosum. BMC Neurosci. 18:31-40.

Lakhani, B., Borich, M.R., Jackson, J.N., Wadden, K.P., Peters, S., Villamayor, A., MacKay, A.L., Vavasour, I.M., Rauscher, A., \& Boyd, L.A. (2016). Motor Skill Acquisition Promotes Human Brain Myelin Plasticity. Neural Plasticity. 2016: 1-7.

Long, P., \& Corfas, G. (2017). Neuroscience. To Learn is to Myelinate. Science 346:298-9.

Micu, I., Plemel, J.R., Lachance, C., Proft, J., Jansen, A.J., Cummins, K., van Minnen, J., Syts, P.K. (2016). The molecular physiology of the axomyelinic synapse. Experimental Neurology. 276: 41-50.

Mount, C.W., Monje, M. (2017). Wrapped to Adapt: Experience-Dependent Myelination. Neuron. 95: 743-56.

Nickel, M., Gu, C. (2018). Regulation of Central Nervous System Myelination in Higher Brain Functions. Neural
Plasticity Volume 2018, Article ID 6436453, 12 pages https://doi.org/10.1155/2018/6436453.

Oberlin, L.E., Verstynen, T.D., Burzynska, A.Z., Voss, M.W., Prakash, R.S., Chaddock-Heyman, L., Wong, C., Fanning, J., Awick, E., Gothe, N., Phillips, S.M., Mailey, E., Ehlers, D., Olson, E., Wojcicki, T., McAuley, E., Kramer, A.F., Erickson, K.I. (2016). White matter microstructure mediates the relationship between cardiorespiratory fitness and spatial working memory in older adults. Neuroimage. 131: 91-101.

Ritchie, S. J., Bastin, M. E., Tucker-Drob, E. M., Maniega, S. M., Engelhardt, L. E., Cox, S.R., Royle, N.A., Gow, A.J., Corley, J., Pattie, A., Taylor, A.M., Hernandez, M.D.C.V., Starr, J.M., Wardlaw, J.M., Deary, I.J. (2015). Coupled changes in brain white matter microstructure and fluid intelligence in later life. J. Neurosci. 35, 8672-8682.

Sampaio-Baptista, C., Johansen-Berg, H. (2017). White Matter Plasticity in the Adult Brain. Neuron. 96: 1239-1251. https://doi.org/10.1016/j.neuron.2017.1 1.026 .

Singh, A., Abraham, W.C. (2017). Astrocytes and synaptic plasticity in health and disease. Exp Brain Res. 235:1645-1655. 
Swire, M., \& Constant, C.F. (2018). Seeing is Believing: Myelin Dynamic in the Adult CNS. Neuron. 98: 684-6.

Thomas, A.G., Dennis, A., Rawlings, N.B., Stagg, C.J., Matthews, L., Morris, M., Kolind, S.H., Foxley, S., Jenkinson, M., Nichols, T.E., Dawes, H., Bandettini, P.A., Johansen-Berg, H. (2016). Multimodal characterization of rapid anterior hippocampal volume increase associated with aerobic exercise. Neuroimage 131, 162-170.

Travaglia, A., Bisaz, R., Cruz, E., Alberini, C.M. (2016). Developmental changes in plasticity, synaptic, glia and connectivity protein levels in rat dorsal hippocampus. Neurobiol Learn Mem. 135: $125-138$.

Wake, H., \& Kato, D. (2015). Brain Function and White Matter. Brain Nerve. 67(4):505-12.

Williamson, J.M., Lyons, D.A. (2018). Myelin Dynamics Throughout Life: An Ever-Changing Landscape? Cellular Neuroscience. 2018: 1-8.
Xiao, L., Ohayon, D., Mckenzie, I. A., Sinclair-Wilson, A., Wright, J. L., Fudge, A. D., Emery, B., Li, H., Richardson, W.D. (2016). Rapid production of new oligodendrocytes is required in the earliest stages of motorskill learning. Nat. Neurosci. 19, 1210 1217. doi:10.1038/nn.4351

Yamazaki, Y. (2019). Oligodendrocyte Physiology Modulating Axonal Excitability and Nerve Conduction. In: Sango K., Yamauchi J., Ogata T., Susuki K. Myelin. Advances in Experimental Medicine and Biology, vol 1190. Springer, Singapore

Yamazaki, Y, Hozumi, Y., Kaneko, K., Fujii, S. (2018). Modulatory Effects of Perineuronal Oligodendrocytes on Neuronal Activity in the Rat Hippocampus. Neurochem Res. 43:2740.

Zheng, J., Sun, X., Ma, C., Li, B., Luo, F. (2019). Voluntary wheel running promotes myelination in the motor cortex through Wnt signaling in mice. Molecular Brain. 12: 85-94. 